there is no carer the patient said knowing someone is voluntarily visiting them they felt valued, less alone.

In a world where care has become increasingly professionalised, volunteers offering home support provide a valuable link between the medical needs of the patient, the physical and emotional needs of carers and patients.

\section{P-167 QUANTITATIVE, QUALITATIVE AND ECONOMIC REVIEW OF MARIE CURIE INTEGRATED PALLIATIVE CARE SERVICE}

${ }^{1}$ Niall Kieran, ${ }^{2}$ Heather Heathfield, ${ }^{1}$ Louise Hall, ${ }^{1}$ Diana Hekerem, ${ }^{3}$ Matthew Armstrong, ${ }^{3}$ Carole Morris. ${ }^{1}$ Marie Curie, Glasgow, UK; ${ }^{2}$ OPM Group; ${ }^{3}$ NHS National Services UK

\subsection{6/bmjspcare-2016-001245.189}

Introduction The pilot service delivered tailored care and support at home for terminally ill people and their families. Locally coordinated support included nursing care, personal care, emotional support and practical information delivered by a team of registered nurses, healthcare assistants, health and personal care assistants and trained volunteers. An anticipated benefit was a changed pattern of use across health and social care, reducing the use of acute services and increasing the number of patients able to die at home.

Aim The evaluation aimed to address patient and carer experience, quality and costs of the service.

Methods A mixed-methods methodology was used to analyse service use and outcomes across the pilot. Stakeholder, healthcare professional and service user surveys and interviews were carried out to create case studies and analyse service quality. Data linkage was carried out between 153 patients supported by the pilot and their matched controls (who died before the start of the pilot service) to assess the impact of the integrated service on hospital use and place of death. Finally, economic analysis used cost per activity from NHS Scotland Health Services to estimate potential savings.

Results The evaluation evidenced improved quality outcomes for patients and a changed pattern of hospital use. Significantly fewer patients in the pilot group experienced a hospital admission (27\%), compared to their matched controls (40\%) with a shorter average stay in hospital (2.5 days less per admission) and more time in the community (15.8\%). Furthermore, significantly more patients in the pilot group $(73.7 \%)$ died at home, compared to their matched controls (29.1\%). The economic analysis showed a potential annual reduction in the costs of end-of-life care in Fife of $£ 182,283$.

Conclusion The integrated service model demonstrates that investment in coordinated palliative care services delivers positive outcomes in quality of care and health economics.

\section{P-168 A REVIEW OF OUT OF HOURS (OOH) CALLS MADE TO A COMMUNITY PALLIATIVE CARE SERVICE}

Helen Brewerton, Martyn Webb. Royal Trinity Hospice, London, UK

\subsection{6/bmjspcare-2016-001245.190}

The National Institute for Health and Clinical Excellence published the 'Quality Standard for End of Life Care for Adults' in 2011 (updated 2013).

Four of the standards make specific reference to provision of a care across the $24 / 7$ period.
Currently all patients under the community palliative care service, their families and any professionals supporting them can access out-of-hours $(\mathrm{OOH})$ specialist palliative care support from a clinical nurse specialist (CNS) via the telephone. It is widely considered that the optimal service provision would include access to face to face visits $24 / 7$.

In order to understand the scope of local need for access to a 24/7 visiting service, a review of all $\mathrm{OOH}$ calls received over a one-month period was undertaken.

A retrospective review was undertaken examining the clinical records relating to $34 \mathrm{OOH}$ calls in an attempt to understand whether an $\mathrm{OOH}$ face to face visit by a CNS would have led to a more favourable patient outcome.

The reviewers felt that on the following four occasions, face to face assessment carried out by a CNS may have led to a more favourable patient outcome:

1. No contact was made with the OOH CNS. Patient was transferred to hospital with no assessed clinical need.

2. Patient had multiple symptoms which made telephone assessment difficult.

3. Patient suffering from breathing difficulties with known social complexities

4. Patient had no recent CNS input and had uncontrolled symptoms.

Next steps include Increased promotion of the OOH CNS service and when and how to access it.

Provision of written guidance for patients and carers about the potential for changes in the patient's condition and instruction on how to proactively manage symptoms to avoid crises.

\section{P-169 WHAT ARE THE PERCEPTIONS OF THE COMMUNITY PALLIATIVE CARE TEAM REGARDING THE CURRENT PROVISION OF TELEPHONE SUPPORT?}

1,2Trudie Potts. 'Birmingham St Mary's Hospice, Birmingham, UK; ${ }^{2}$ De Montfort University, Leicester

10.1136/bmjspcare-2016-001245.191

Aim The aim of this study is firstly to understand the form and content of telephone support delivered by the community palliative care team (single inner-city hospice). Secondly, to explore the perspectives of the team members, regarding their lived experience of providing telephone support.

Background The role of the community clinical nurse specialist in palliative care, is pivotal to supporting patients with complex needs. There is limited literature acknowledging and quantifying, the clinical and supportive role of day-to-day telephone advice, provided by the team.

Method A two phase method was adopted. Firstly, a contact analysis table, logging incoming calls and messages received by the team, provided a picture of the current provision of telephone advice. Secondly, adopting a phenomenological approach, indepth interviews enabled the researcher, to explore the perceptions of the community palliative care team, on the current provision of telephone support.

Findings Relatives of patients utilise the telephone advice from the CNSs, for both symptom management and emotional support. The average length of a call for a CNS is eight minutes and two minutes for the administration team. The CPCT perceive that the availability and accessibility for advice over the telephone 